\title{
ARTÍCULOS
}

\section{SUJETOS Y CERTIDUMBRES NEOLIBERALES. UN ANÁLISIS DE LA ARTICULACIÓN ENTRE LAS LEYES DE REFORMA LABORAL, DE APOYO AL EMPRENDEDOR Y LA LOMCE*}

\author{
NEOLIBERAL SUBJECTS AND CERTITUDES. A STUDY ON LINKAGES \\ BETWEEN LABOR LAWS, ENTREPRENEURSHIP AND EDUCATIONAL \\ LEGISLATION
}

\author{
Ángel Borruel Armendariz ${ }^{1}$ \\ Instituto Madrileño de Antropología \\ Gaël Carrero Gros ${ }^{2}$ \\ Universidad Autónoma de Madrid
}

\section{Adela Franzé Mudanó ${ }^{3}$}

Universidad Complutense de Madrid

Sergio García García ${ }^{4}$

Universidad Complutense de Madrid

\author{
Gladys Nieto Martínez ${ }^{6}$ \\ Universidad Autónoma de Madrid
}

Daniel Parajuá Navarrete ${ }^{7}$

Universidad Complutense de Madrid

\author{
Álvaro Pazos Garciandía ${ }^{8}$ \\ Universidad Autónoma de Madrid \\ Pilar Rodrigo Álvarez ${ }^{9}$ \\ Instituto Madrileño de Antropología
}

Erika García González ${ }^{5}$

Universidad Complutense de Madrid

\footnotetext{
* Este artículo ha sido elaborado por los miembros del Grupo Temático Antropología y Derechos Humanos del Instituto Madrileño de Antropología. Es una ampliación de trabajos presentados previamente en las III Jornadas del Instituto Madrileño de Antropología, en mayo del 2014, en la Jornada "Certidumbres neoliberales" en el Centro Social La Caba, de Madrid, el 25 de octubre de 2014, y en el Conversatorio "Laboratorios de lo social. Experiencias alternativas y potencialidades críticas", organizado por el Grupo Temático de Antropología y Derechos Humanos del Instituto Madrileño de Antropología, celebrado el 28 de noviembre de 2015 en Madrid.

1 Correo electrónico: angelborruel@gmail.com. ORCID iD: <https://orcid.org/0000-0003-1319-4804>.

2 Correo electrónico: gael.carrero@uam.es. ORCID iD: <https://orcid.org/0000-0002-1100-0802>.

3 Correo electrónico: adelafranze@ucm.es. ORCID iD: <https://orcid.org/0000-0002-2991-4434>.

4 Correo electrónico: sergig07@ucm.es. ORCID iD: <https://orcid.org/0000-0002-9551-429>.

5 Correo electrónico: erikagar@ucm.es. ORCID iD: <https://orcid.org/0000-0002-9003-22899>.

6 Correo electrónico: gladys.nieto@uam.es. ORCID iD: <https://orcid.org/0000-0001-6674-7105>.

7 Correo electrónico: dparajua@pdi.ucm.es. ORCID iD: <https://orcid.org/0000-0001-9679-4412>.

8 Correo electrónico: alvaro.pazos@uam.es. ORCID iD: <https://orcid.org/0000-0002-8376-3083>.

9 Correo electrónico: prodrigoa@outlook.es. ORCID iD: <https://orcid.org/0000-0002-7656-2347>.
} 
Cómo citar este artículo / Citation: Borruel Armendariz, Ángel, Gaël Carrero Gros, Adela Franzé Mudanó, Sergio García García, Erika García González, Gladys Nieto Martínez, Daniel Parajuá Navarrete, Álvaro Pazos Garciandía y Pilar Rodrigo Álvarez. 2021. "Sujetos y certidumbres neoliberales. Un análisis de la articulación entre las leyes de reforma laboral, de apoyo al emprendedor y la LOMCE". Disparidades. Revista de Antropología 76(2): e020. doi: <https://doi.org/10.3989/dra.2021.020>.

RESUMEN: En este artículo nos interesamos por algunos cambios profundos que están afectando a los derechos sociales en España, en el contexto neoliberal contemporáneo. Más concretamente, abordamos la comparación de tres cuerpos de leyes recientes (de reforma laboral, de apoyo al emprendedor y de educación), y analizamos cómo se presentan en ellas sujetos de derecho en relación al trabajo y la formación. El estudio destaca una articulación de los diferentes textos y constata, de un lado, la transformación del sujeto trabajador en sujeto empleable, que se corresponde con la figura de subjetivación del emprendedor. De otro lado, muestra cómo la LOMCE dibuja de manera más nítida este sujeto, y precisa los procedimientos para su fabricación.

PALABRAS CLAVE: Derechos sociales; Neoliberalismo; Subjetivación; Trabajo; Educación; Emprendimiento.

ABSTRACT: In this paper, we are interested in some deep changes affecting social rights in Spain, in the current neoliberal world context. Specifically, we address a comparison of three legal areas (labour reform, entrepreneurship and educational) analyzing how people are defined in connection with rights, work and education. This survey underlines the linkage of three different legal texts and, on one side verifies the transformation from workers to employable people, which is connected to the neoliberal subjectivation figure of entrepreneur; and on the other side, we show how the current educational law (LOMCE) defines this figure in a clear-cut way, specifying its production processes.

KEYWORDS: Social Rights; Neoliberalism; Subjectivation; Labour; Education; Entrepreneurship.

Copyright: @ 2021 CSIC. Este es un artículo de acceso abierto distribuido bajo los términos de la licencia de uso y distribución Creative Commons Reconocimiento 4.0 Internacional (CC BY 4.0).

\section{INTRODUCCIÓN}

En 2014 comenzamos una investigación que buscaba analizar las retóricas de las nuevas leyes de reforma laboral, de apoyo a los emprendedores y de educación que fueron lanzándose sucesivamente en los últimos años en España con la implementación de políticas neoliberales ${ }^{10}$. Entendemos que las medidas en los ámbitos laboral, de empresas y de la educación, que se dictan en estos tres cuerpos legislativos están relacionadas entre sí, y que esta conexión se hace inteligible en cuanto colocamos las leyes en el contexto del desarrollo modernizador y neoliberal en el que la sociedad española está embarcada. Cuando hablamos de "neoliberalismo", nos referimos a la crisis del Estado de Bienestar y la implementación de un nuevo modo de regulación del capitalismo (Harvey 1998), pero sobre todo a la conformación,

10 Ley $3 / 2012$ de 6 de julio de medidas urgentes para la reforma del mercado laboral; Ley 14/2013 de 27 de septiembre, de apoyo a los emprendedores y su internacionalización; Real Decreto 4/2013 de 22 de febrero, de medidas de apoyo al emprendedor y de estímulo del crecimiento y de la creación de empleo; Ley Orgánica 8/2013 de 9 de diciembre para la mejora de la calidad educativa. asociada a ello, de una racionalidad y un régimen discursivo nuevos, una "forma de la existencia" con implicaciones sociales y subjetivas generales (Laval y Dardot 2013; Brown 2015). Nuestro interés está puesto precisamente en apuntar el modo como los textos de las leyes contribuyen a definir y, por su poder performativo específico, a producir ciertas transformaciones sociales y subjetivas; y cómo sus retóricas toman sentido dentro de aquel régimen discursivo.

De manera sucinta, las transformaciones neoliberales en cuestión constituyen un proceso de individualización de las formas sociales y de los sujetos, la realización de lo que Pierre Bourdieu ha llamado un "programa de destrucción metódica de los colectivos» encaminado a imponer la utopía neoliberal del sistema económico autorregulado (1998). Este proceso tiene unas implicaciones importantísimas en el plano político y de las libertades, pues supone una transformación "des-politizadora», cuando no una erosión radical, de las nociones mismas de ciudadanía y democracia (Castel 2003, 2010; Brown 2015). Analizamos esto justamente en los textos de las leyes que hemos revisado: en ellas se cuestionan algunos derechos sociales históricamente adquiridos por colectivos sociales como los 
trabajadores; y se apuntala un nuevo sujeto de derechos, privado, definido solo por rasgos, disposiciones o capacidades puramente individuales (Dardot y Laval 2013). Se trata de una crisis y transformación de las formas y subjetivaciones políticas, que se desarrolla entreverada con una crisis y transformación de los modos de trabajo, y de relación con el trabajo (Balazs y Pialoux 1996).

Efectivamente, en el contenido discursivo de estas regulaciones observamos que el trabajador se desdibujaba como sujeto de derechos, así como parecía delinearse un nuevo sujeto en la figura del emprendedor. Este aparecía como un agente capaz de activarse, generar su propio empleo, proveerse de servicios sociales básicos -en educación y sanidad- en un contexto neoliberal en el que el Estado ya no garantizaría tales derechos. Así, de la pérdida de los derechos laborales -protección social o la negociación colectiva- resultaba la apelación al emprendimiento y la formación como únicos derechos. En esta nueva etapa el antiguo trabajador se convierte en un empresario sin empresa, o cuya empresa es él mismo. De allí que los futuros emprendedores deban adquirir nuevos valores desde edades tempranas, derivados de ese "espíritu» de desarrollo del talento y la iniciativa. Los discursos del emprendimiento conectan con la «nueva razón del mundo" (Laval y Dardot 2013) que sitúa la competencia como norma universal y de subjetivación.

En este trabajo avanzaremos en un recorrido analítico por las normas legislativas antes mencionadas, a través de sus retóricas y los dispositivos que contribuyen a producir estos nuevos sujetos empleables. El análisis de discurso que hemos llevado a cabo se basa en un enfoque pragmático, que entiende los discursos como prácticas que construyen o articulan performativamente realidad. Atendemos, por ello, más a las estrategias retóricas que a las lógicas gramaticales de estos corpus, en la línea de aproximación al poder discursivo de Bourdieu (1985) y de Foucault (1969), y abordamos en consecuencia, las leyes analizadas centrándonos en las "teorías sociales» subyacentes y los modelos cognitivos y prácticos que contribuyen a asentar (en la perspectiva de antropólogos que han estudiado los derechos, como Cowan, Dembour y Wilson 2001; o Cowan 2010). En síntesis, nuestro trabajo analítico ha tratado de detectar los marcos y esquemas, las categorías y los modos de razonamiento con los que estos discursos legales definen, y contribuyen a imponer una realidad o situación como dada, un tipo de su- jetos, y una problemática específica a la que la propia legislación responde.

\section{DEL SUJETO TRABAJADOR AL SUJETO EMPLEABLE}

En la Ley de reforma laboral se propone la desestructuración o desarticulación de la noción de trabajo y del sujeto trabajador, como sujeto colectivo, lo que hace esta ley tan problemática desde el punto de vista de los derechos ${ }^{11}$. La desarticulación del sujeto trabajador se aprecia en el desarrollo de diversas estrategias retóricas:

La primera de estas estrategias es el planteamiento de la crisis como una realidad des-historizada y naturalizada que se manifiesta básicamente en el fuerte crecimiento del desempleo, y que es, además, la expresión de un problema que se considera meramente técnico: las "debilidades del modelo laboral español".

La crisis económica que atraviesa España desde 2008 ha puesto de relieve las debilidades del modelo laboral español. La gravedad de la crisis actual no tiene precedentes. España ha destruido más empleo, y más rápidamente, que las principales economías europeas. Los datos de la última Encuesta de Población Activa describen bien esta situación: la cifra de paro se sitúa en 5.273.600 personas, con un incremento de 295.300 en el cuarto trimestre de 2011 y de 577.000 respecto al cuarto trimestre de 2010. La tasa de paro sube en 1,33 puntos respecto al tercer trimestre y se sitúa en el $22,85 \%$. (Ley $3 / 2012$ de 6 de julio. Preámbulo. Parte I)

Se supone también, una noción técnica de aquel «modelo», que no considera, en consecuencia, las razones de ser y la historia a la que responden las definiciones de relaciones laborales, los derechos y garantías que lo componen, como si se tratara de un instrumento implementado en un determinado momento para alcanzar un objetivo (el empleo), y que se manifestara ahora como obstáculo.

Aunque se plantee la crisis como "sin precedentes», y la Ley como «medida urgente» y como una respuesta ante una determinada situación, los problemas son definidos sin embargo como "estructurales», y la reforma necesaria como «de envergadura» porque

11 Un análisis más extenso de la Ley de Reforma Laboral, que desarrolla los ejes que apuntamos aquí, es el de Serrano, Rodríguez y Artiaga (2014). 
lo que la crisis evidencia es, en realidad, la situación límite a la que ha llegado algo que es más antiguo y que, desde un principio presentaría problemas: el propio modelo laboral español. Se plantea, entonces, una intervención sobre este.

La crisis económica ha puesto en evidencia la insostenibilidad del modelo laboral español. Los problemas del mercado de trabajo lejos de ser coyunturales son estructurales, afectan a los fundamentos mismos de nuestro modelo sociolaboral y requieren una reforma de envergadura que, pese a los cambios normativos experimentados en los últimos años continúa siendo reclamada por todas las instituciones económicas mundiales y europeas que han analizado nuestra situación, por los mercados internacionales que contemplan la situación de nuestro mercado de trabajo con enorme desasosiego y, sobre todo, por los datos de nuestra realidad laboral, que esconden verdaderos dramas humanos. Las cifras expuestas ponen de manifiesto que las reformas laborales realizadas en los últimos años, aún bienintencionadas y orientadas en la buena dirección, han sido reformas fallidas. (Ley 3/2012 de 6 de julio, Preámbulo. Parte I)

En segundo lugar, se plantea una estrategia retórica que hace una lectura puramente técnica e instrumental de las instituciones laborales, los trámites administrativos y los procedimientos judiciales, descontextualizándolos históricamente, haciéndoles perder su sentido, mostrándolos solo como obstáculos o rigideces. Se proponen así en el Capítulo IV del Preámbulo de la Ley $3 / 2012$ de 6 de julio, una serie de medidas encaminadas a diluir, simplificar o agilizar esas dificultades:

1) «el sistema de clasificación profesional pasa a tener como la única referencia el grupo profesional con el objetivo de sortear la rigidez de la noción de categoría profesional y hacer de la movilidad funcional ordinaria un mecanismo de adaptación más viable y eficaz».

2) «Se simplifica la distinción entre modificaciones sustanciales individuales y colectivas, se incluye la modificación sustancial de funciones y de estructura y cuantía salarial como causa de extinción voluntaria del contrato de trabajo con derecho a indemnización y, la modificación de condiciones de trabajo recogidas en convenio colectivo del Título III del Estatuto de los Trabajadores se reconducen al apartado 3 artículo 82 del Estatuto de los Trabajadores».
3) «En materia de suspensión del contrato de trabajo y reducción de la jornada por causas económicas, técnicas, organizativas o productivas, la presente Ley pretende afianzar este mecanismo alternativo a los despidos, dándole agilidad mediante la supresión del requisito de autorización administrativa y estableciendo una prórroga del sistema de bonificaciones y reposición de prestaciones por desempleo previsto para estos supuestos».

4) «En materia de negociación colectiva se prevé la posibilidad de descuelgue respecto del convenio colectivo en vigor, se da prioridad al convenio colectivo de empresa y se regula el régimen de ultractividad de los convenios colectivos. Las modificaciones operadas en estas materias responden al objetivo de procurar que la negociación colectiva sea un instrumento, y no un obstáculo, para adaptar las condiciones laborales a las concretas circunstancias de la empresa».

Una tercera estrategia retórica, en cierto modo consecuencia de las anteriores, es el cuestionamiento de las categorías y las nociones colectivas que garantizarían el estatuto de los sujetos de derechos, proyectando ahora una visión negativa sobre estas por la "rigidez» que suponen, por ser una fuente de "conflictividad". Se hace una continua apelación a la individualidad de las relaciones, primero desplazando el marco de negociación desde instituciones y escenarios colectivos hacia el marco particular de la empresa que destaca como la entidad fundamental. Y después, en el interior del ámbito empresarial, se apunta a relaciones individuales entre un empleador y un empleado singulares. Se presenta pues la negociación colectiva como obstáculo, en relación a «las circunstancias concretas que atraviesan las empresas»:

\footnotetext{
En materia de negociación colectiva se prevé la posibilidad de descuelgue respecto del convenio colectivo en vigor, se da prioridad al convenio colectivo de empresa (...) Que la negociación colectiva sea un instrumento y no un obstáculo, para adaptar las condiciones laborales a las concretas circunstancias de las empresas. (Ley 3/2012 de 6 de julio. Preámbulo, Parte IV)
}

Se plantea así la necesidad de "descentralizar" la negociación colectiva para considerarla como un mero instrumento:

La novedad que ahora se incorpora va encaminada, precisamente, a garantizar dicha descentralización convencional en aras de facilitar una negociación 
de las condiciones laborales en el nivel más cercano y adecuado a la realidad de las empresas y de sus trabajadores. (Ley $3 / 2012$ de Julio. Preámbulo, Parte IV)

La propia Ley explicita que su objetivo es facilitar, reforzar y apoyar la flexibilidad (de las empresas) sin dejar de garantizar los derechos o la seguridad de los trabajadores, apelando para ello al concepto de «flexiseguridad ${ }^{12}$. En realidad, el desarrollo de este concepto en la Ley reconoce por igual inicialmente dos intereses contrastados según sean empresarios y trabajadores: flexibilidad y seguridad, respectivamente. Se invisibiliza así el conflicto que existe entre ambos, insistiendo en las ideas de equilibrio y convergencia.

La reforma propuesta trata de garantizar tanto la flexibilidad de los empresarios en la gestión de los recursos humanos de la empresa como la seguridad de los trabajadores en el empleo y adecuados niveles de protección social. Esta es una reforma en la que todos ganan, empresarios y trabajadores, y que pretende satisfacer más y mejor los intereses de todos. (Ley 3/2012 de 6 de julio. Preámbulo, Parte I).

Sin embargo, en el desarrollo de la Ley (que pone el acento en las necesidades cambiantes de las empresas, y en la eliminación de reglamentaciones preestablecidas e intermediarios) prima la noción de flexibilidad frente a la de seguridad. De aquí se sigue que solo uno de los dos agentes en principio reconocidos (empresarios, capital), permanece como sujeto en tanto que el otro (trabajadores, trabajo) es negado como tal. Por lo que la Ley de reforma del mercado laboral resulta ser, entre otras cosas, un mecanismo de destrucción del trabajo como fuente de derechos, y del trabajador como sujeto de los mismos.

Esta primacía de la flexibilidad y la invisibilización del sujeto trabajador se declina en una serie de procedimientos de acción que las concretan:

La reforma apuesta por el equilibrio en la regulación de nuestras relaciones de trabajo: equilibrio entre la flexibilidad interna y la externa; entre la regulación de la contratación indefinida y la temporal, la de la movilidad interna en la empresa y la de los mecanismos extintivos del contrato de trabajo; entre

12 Una historia de la imposición de este concepto en España, y un análisis centrado en la legislación en materia de relaciones laborales, se desarrolla en Fernández y Serrano, 2014. las tutelas que operan en el contrato de trabajo y las que operan en el mercado de trabajo, etc. El objetivo es la flexiseguridad. Con esta finalidad, la presente Ley recoge un conjunto coherente de medidas que pretenden fomentar la empleabilidad de los trabajadores, reformando aspectos relativos a la intermediación laboral y a la formación profesional (capítulo I); fomentar la contratación indefinida y otras formas de trabajo, con especial hincapié en promover la contratación por PYMES y de jóvenes (capítulo II); incentivar la flexibilidad interna en la empresa como medida alternativa a la destrucción de empleo (capítulo III); y, finalmente, favorecer la eficiencia del mercado de trabajo como elemento vinculado a la reducción de la dualidad laboral, con medidas que afectan principalmente a la extinción de contratos de trabajo (capítulo IV). (Ley 3/2012 de 6 de julio. Preámbulo, Parte II)

Lo que aparece con claridad en este desglose es la noción de empleabilidad y con ella, se vislumbra el surgimiento de un tipo de sujeto empleable que sustituye al sujeto trabajador. La definición de un sujeto empleable no se refiere, en rigor, a un sujeto social, sino a un conjunto de competencias y habilidades, a una entidad impersonal y sin contenido social, que se define solo por la capacidad individual de adaptación, supeditada a la racionalidad instrumental de las empresas. En este sentido, el paso de uno a otro tipo de sujeto supone un proceso de individualización de los agentes sociales en el discurso legislativo. En definitiva, el sujeto empleable es un individuo, con todo lo que esto implica de naturalización de las capacidades, y de asunción personal de la responsabilidad sobre el desarrollo y sobre la puesta en valor de lo que se empieza a conceptualizar en las leyes como «talento».

Paralelamente se asiste, por un lado, a un vaciamiento y cuestionamiento de los diferentes contenidos de los derechos grupales (negociación colectiva y convenios, sistemas de protección laboral, etc.), y, por otro, a un desplazamiento del foco desde estos hacia derechos individuales (formación, modalidades interpersonales de contratación, flexibilización de los horarios y salarios, posibilidad de deslocalización de los espacios de trabajo, etc.).

\section{DEL SUJETO EMPLEABLE AL SUJETO EMPRENDEDOR}

En 2013 se promulgan dos leyes, una de medidas de apoyo al emprendedor y de estímulo del crecimiento y la creación de empleo (RD-L 4/2013 de 22 de febrero) 
y otra, de apoyo a los emprendedores y su internacionalización (Ley 14/2013 de 27 de septiembre). Estas leyes, muy conectadas entre sí, están a su vez vinculadas muy estrechamente con la Ley de reforma laboral. Son, como esta última, leyes sobre el empleo, el mercado laboral y el modelo de relaciones laborales. Sin embargo, en su retórica las nociones de «trabajo» y «trabajadores» han desaparecido ya por completo, ni siquiera se mencionan; por el contrario, se repiten las de "empleabilidad» y "autoempleo». Autoempleados (empresarios) y empleables vienen a ser dos figuras de un nuevo tipo de sujeto: el emprendedor ${ }^{13}$.

Al igual que ocurría en la Ley de la reforma laboral, la problemática a la que se trata de responder con estas leyes se sitúa en el contexto de la crisis, presentada como causa y justificación de las propuestas que ofrecen. En contraste con el modo en que lo hacía la de reforma, en la que se planteaba la crisis como una realidad natural que, como tal, precisa de medidas técnicas, en las leyes del emprendimiento la crisis se enlaza con una «crisis de valores». Para responder a la crisis así definida, se proponen no solo medidas de corte económico, sino medidas que apuntan a construir y afianzar este nuevo tipo de sujeto, con una «cultura», un "espíritu», una «mentalidad». Es decir, con unos atributos determinados que es preciso desarrollar, que se concretan a través de medidas que tratan de impulsar y reforzar la "actividad emprendedora», la cual se traduce en términos de capacidad de «asunción de riesgos ${ }^{14}$.

Para invertir esta situación, es necesario un cambio de mentalidad en el que la sociedad valore más la actividad emprendedora y la asunción de riesgos. La piedra angular para que este cambio tenga lugar es, sin duda, el sistema educativo. (Ley 14/2013 de 27 de septiembre; Preámbulo I)

De esta forma desaparece el contexto político de precariedad laboral -de asimetrías de poder, conflicto y vulnerabilidad social- en el que se produce realmente la crisis, y es reemplazado por un ámbito social

13 Sobre esta relación, y la invención de la categoría de "emprendedor», trata Serrano (2016).

14 Todos los temas propios del discurso y el imaginario del management (emprendizaje, riesgo, creatividad, competencia, innovación, etc.), que Boltanski y Chiapello (2002) y Fernández (2007) han analizado, han venido impregnando cada vez más ámbitos de la vida social y marcando formas de subjetividad generalizadas (Alonso y Fernández 2013, 2018). neutro en el que actúa un tipo de sujeto que emprende y que asume riesgos. Así definido el problema, se apunta a un elemento importante, que conecta en una lógica común la Ley de reforma laboral, las leyes de apoyo a emprendedores, y la futura Ley de Educación: la difusión y socialización en la «cultura emprendedora», es decir, en las «competencias y habilidades requeridas para emprender».

El nuevo sujeto que se trata de formar y reforzar es el "emprendedor». Esta categoría, tal y como es utilizada en la ley, se define en términos psicológicos y morales, es decir, remite a rasgos individuales de carácter, personalidad y comportamiento. Se trata, como explicita la propia Ley $14 / 2013$, del «espíritu emprendedor».

1-Los currículos de Educación Primaria, Secundaria Obligatoria, Bachillerato y Formación Profesional incorporarán objetivos, competencias, contenidos y criterios de evaluación de la formación orientados al desarrollo y afianzamiento del espíritu emprendedor, a la adquisición de competencias para la creación y desarrollo de los diversos modelos de empresas y al fomento de la igualdad de oportunidades y del respeto al emprendedor y al empresario, así como a la ética empresarial.

2-Las Administraciones educativas fomentarán las medidas para que el alumnado participe en actividades que le permita afianzar el espíritu emprendedor y la iniciativa empresarial a partir de aptitudes como la creatividad, la iniciativa, el trabajo en equipo, la confianza en uno mismo y el sentido crítico. (Ley 14/ 2013, Título I, Capítulo I, Artículo 4)

La retórica del emprendimiento, que identificamos en estas leyes, forma parte, en realidad, de una doxa o un conjunto de verdades de sentido común sobre la persona y la sociedad, ampliamente aceptadas como evidentes, no cuestionadas, en distintos ámbitos de la vida social actual. Es un lenguaje psicologicista que en lugar de hablar de condiciones y de características sociales de los sujetos, de los ciudadanos, etc., habla de rasgos individuales como la creatividad, la iniciativa, la confianza en uno mismo, el talento, la inversión en talento, la valorización de sí mismo, el espíritu innovador, el desarrollo personal identificado con el profesional, etc. (Serrano 2016).

Todas las medidas educativas que contemplan las leyes del apoyo al emprendimiento, apelan a su materialización y desarrollo más pormenorizado en la futura Ley Orgánica para la Mejora de la Calidad Educativa (LOMCE): 
La idea del emprendedor y de la cultura empresarial como elementos indispensables para el desarrollo económico y social de España se ha venido introduciendo de forma expresa en algunas etapas educativas, pero se considera necesario subrayar y ampliar este elemento, incorporándolo como objetivo específico en todas las etapas de la educación básica, así como en la Formación Profesional y en el bachillerato. Consecuentemente, se encomienda a las administraciones educativas la revisión y adecuación de los currículos de las enseñanzas regladas a estos nuevos objetivos. [...] Además, las medidas que esta Ley introduce en el ámbito educativo deben completarse con las de la Ley Orgánica para la Mejora de la Calidad Educativa, que prevé la estimulación del espíritu emprendedor a través de la posibilidad de elección de las trayectorias educativas más adecuadas y de la creación de las condiciones para que todos los alumnos puedan adquirir y expresar sus talentos. (Ley 14/ 2013, Título I, Capítulo I)

Como veremos a continuación la LOMCE va a responder a estas apelaciones, desarrollando todo un conjunto de medidas y diseñando lo que podríamos considerar un plan global de formación de sujetos emprendedores.

\section{HACIA EL SUJETO EDUCABLE}

Si la Ley de reforma laboral se destina a la disolución del sujeto como entidad colectiva -trabajador-, y por ende de derechos, y las dos subsiguientes anuncian el neo sujeto "emprendedor», la LOMCE se consagra a su definición, así como a la transformación del espacio educativo en tanto lugar de su promoción y fabricación. En el corazón de la reforma educativa está la empleabilidad, la necesidad de encajar la formación en el sistema productivo y, a la vez, que los nuevos sujetos resultantes del proceso formativo adquieran, incorporen y activen disposiciones de empleabilidad y emprendimiento.

Nos interesamos, en lo que sigue, por dos líneas de análisis: una que atiende a las diversas estrategias retóricas que, en el campo educativo, extienden y continúan lo establecido en otros ámbitos, como el laboral. Y otra línea, que muestra algunos de los dispositivos específicos del campo educativo de producción de esos sujetos empleables y emprendedores.

\subsection{RETÓRICAS DE LA LOMCE}

En primer lugar, se despliega un conjunto de estrategias retóricas congruentes con las de las leyes ante- riores -del 2012 y 2013-. De modo semejante a la Ley de reforma laboral, la LOMCE esboza un escenario actual y futuro de profundas transformaciones, de las que se predica un carácter «cada vez más global y exigente» (Ley Orgánica 8/2013. Preámbulo, III). Si bien ha desaparecido la mención a la crisis como elemento sobre el cual pivota la argumentación legislativa, en su lugar la reforma se reinscribe en una realidad igualmente des-historizada y autoevidente, a la que se presenta bajo la imagen de inapelable dinamismo -«más abierta, global y participativa»- que requiere «nuevos perfiles de ciudadanos y trabajadores, más sofisticados y diversificados [...] capaces de asumir que la verdadera fortaleza está en la mezcla de competencias y conocimientos diversos» (Ley Orgánica 8/2013. Preámbulo, IV).

En un escenario así planteado, la reforma emerge como solución de carácter técnico al desajuste de un modelo, el educativo. La LOMCE muestra un escenario social y económico en el que el sistema educativo está dominado por el fracaso escolar. Los datos de los informes PISA ${ }^{15}$ sirven para argumentar el estancamiento que sufre el sistema educativo español que no consigue superar los últimos puestos en el ranking respecto a otros países europeos, a la vez que enfatiza la imperiosa necesidad de su adecuación a los estándares de calidad de la Unión Europea. Apoyándose en fuentes autorizadas, que transfieren legitimidad a los propios planteamientos mediante el recurso al discurso experto, importante (Bourdieu 1985), este discurso se afianza mediante el uso estratégico de datos estadísticos, que objetivan el fracaso al margen de su producción social. Contrariamente a las débiles fundamentaciones dedicadas a otros temas, estos datos aportan toda la contundencia de la prueba, y contribuyen asimismo a construir la veracidad de la relación directa y unívoca, establecida por el texto de la ley, entre las elevadas tasas de abandono escolar temprano, el reducido número de estudiantes que alcanza la excelencia y los persistentes desajustes del sistema a los que se responsabiliza de ello.

Esta visión movilizada por la LOMCE se despliega al menos en dos argumentos justificativos. De un lado, a aquella imagen de transformación y fluidez sobre la que se construye la economía, se opone el lenguaje de la «rigidez» con el que se caracteriza al sistema educativo. Así, al discurso del cambio que hace de la

15 Programme for International Student Assessment 
economía una fuerza naturalizada e incuestionable, se contrapone el de la institución como una suerte de artefacto que actúa como obstáculo o barrera, evocando la idea corriente que la piensa en términos de coerción y freno a la expansión de las potencialidades y aspiraciones individuales. Este "orden del mundo», por tanto, requiere que se creen mecanismos para solucionar tal problema.

De otro lado, se apoya en el vaciamiento de sentido de los debates sobre el sistema educativo. Al calificarlos de «ideológicos» y concebirlos como otros tantos obstáculos de los que se debe «huir» para «avanzar», la retórica de la LOMCE, en última instancia, se sostiene sobre el esquema de la neutralidad, objetividad y racionalidad tecnocrática. Se presenta como una forma de intervención "sensata» y "práctica», ajena a toda sospecha de parcialidad o sesgo, al priorizar, por el contrario, los problemas «concretos»: la lucha contra el abandono temprano y el bajo nivel de calidad formativa, que desembocan en la "lacra» del desempleo, así como en las limitadas posibilidades de «movilidad social».

El sistema actual no permite progresar hacia una mejora de la calidad educativa, como ponen en evidencia los resultados obtenidos por los alumnos y alumnas en las pruebas de evaluación internacionales como PISA (Programme for International Student Assessment), las elevadas tasas de abandono temprano de la educación y la formación, y el reducido número de estudiantes que alcanza la excelencia. La objetividad de los estudios comparativos internacionales, que reflejan como mínimo el estancamiento del sistema, llevan a la conclusión de que es necesaria una reforma del sistema educativo que huya de los debates ideológicos que han dificultado el avance en los últimos años. Es necesaria una reforma sensata, práctica, que permita desarrollar al máximo el potencial de cada alumno o alumna. (Ley Orgánica 8/2013, Preámbulo, V)

En este swtido, como hemos señalado, la Ley de reforma laboral y la LOMCE construyen un escenario social apelando a estrategias semejantes, despolitizadoras. Ambas conforman su retórica sobre una representación autonomizada del orden económico, independiente de la acción y de la moral, y al mismo tiempo construyen a las instituciones como el lugar en el que sí se puede, y debe, intervenir: las exigencias del mercado deben resolverse técnicamente en la esfera propiamente social y humana, para corregir el rumbo de la institución educativa.
En segundo lugar, tal como ocurre con las leyes de apoyo al emprendimiento, la LOMCE produce un desplazamiento en el régimen retórico de justificación, de la institución al individuo; del rol a las actitudes, motivaciones y valores del sujeto (Dubet 2008).

En efecto, desde los primeros párrafos se advierte en el texto legal una retórica individualizante del sujeto y en consecuencia de su relación con el conocimiento y consigo mismo, lo que sirve de fundamento, a su vez, al replanteamiento del papel a jugar por el sistema educativo en este contexto.

La configuración del sujeto de la que se parte lo desocializa y desculturaliza, reduciéndolo a una amalgama de cualidades y valores que le serían inherentes. La construcción (re)individualizante se sostiene y pone de manifiesto en diversas estrategias retóricas, tanto en lo que olvidan como en lo que subrayan. De un lado, se pone en evidencia en la omisión de cualquier referencia a las condiciones sociales de los alumnos que afectan a sus trayectorias, tanto como a las oportunidades de acceso al capital escolar y/o al mercado laboral. De otro, la reiteración de adjetivos posesivos singulariza rasgos que se predican del sujeto, destacando, además, aquellos que el sentido común asocia a la persona (sus capacidades, habilidades, expectativas, vocación, esfuerzos etc.). Así, los sueños, motivaciones, aspiraciones, objetivos, habilidades, etc. del alumno resultan ser la expresión del "talento», el cual, en la medida en que se niega su sociogénesis, se reafirma como capital -naturalizadodel individuo.

La LOMCE no renuncia a asentar un modelo educativo universal e inclusivo; de hecho no solo lo afirma, sino que si algo destaca del sistema español es haber conseguido la universalización de la educación y la escolarización plena. Pero enfatiza, a la vez, un déficit, al parecer acumulado desde tiempo atrás: su leitmotiv es el compromiso con una educación de calidad.

El reto de una sociedad democrática es crear las condiciones para que todos los alumnos puedan adquirir y expresar sus talentos, en definitiva el compromiso con una educación de calidad como soporte de la igualdad y la justicia social. (Ley Orgánica 8/2013, Preámbulo I)

Las rigideces del sistema conducen a la exclusión de los alumnos cuyas expectativas no se adecuan al marco establecido. En cambio, la posibilidad de elegir entre distintas trayectorias les garantiza [...] mayores posibilidades para su desarrollo personal 
y profesional [...] de forma que cada estudiante pueda desarrollar todo su potencial. (Ley Orgánica 8/2013, Preámbulo X)

Estos párrafos ilustran diversas cuestiones de interés. Por una parte, el discurso de la LOMCE tiene relación con la idea del individuo como ser autónomo, autosuficiente e independiente: un individuo que en sociedad funciona por debajo de sus potencialidades y deseos, porque se limita su libertad de elección (Dumont 1970; Castel 2010). Igualmente, moviliza un lenguaje que recupera preocupaciones críticas sobre el espacio educativo, y se apoya en un conjunto de justificaciones compartidas concitando la adhesión del lector por su carácter neo-humanista: contiene evocaciones a una educación universal e inclusiva, individualizada o personalizada, así como a valores de reconocimiento de la diversidad, desarrollo personal, justicia y equidad.

Por otro lado, como venimos sugiriendo, la LOMCE dialoga con las filosofías educativas y marcos normativos anteriores proyectando sobre ellos, más o menos expresamente, una imagen negativa de sus resultados: se les reprocha la "rigidez» que ha desembocado en desiguales oportunidades en el mercado laboral y en la obstaculización de los desarrollos personales. Indudablemente la Ley se hace eco de algunos de los cuestionamientos más asentados en la comunidad educativa en la última década: invoca los argumentos que achacan al modelo comprehensivo de enseñanza la retención "forzada» -en un sistema único y obligatorio hasta los 16 años- de alumnos y alumnas cuyos intereses y expectativas no se adecúan a ello, con el consecuente retraso de los buenos alumnos o el descenso del nivel que esto conllevaría.

Por ello, aunque de manera más implícita y significativa, apunta críticamente al modelo de igualdad y justicia que atribuye a dichos marcos. La Ley escoge estratégicamente las nociones de "equidad» y "calidad» para redefinir la «igualdad» y el «derecho» a la educación.

Equidad y calidad son dos caras de una misma moneda. No es imaginable un sistema educativo de calidad en el que no sea una prioridad eliminar cualquier atisbo de desigualdad. No hay mayor falta de equidad que la de un sistema que iguale en la desidia y en la mediocridad. Para la sociedad española no basta con la escolarización para atender al derecho a la educación, la calidad es un elemento constituyente del derecho a la educación. (Ley Orgánica 8/2013, Preámbulo III)
"Se hace necesario generar la convicción de que el sistema educativo recompensa de manera transparente y equitativa el rendimiento que se logre en los objetivos educativos [...]. (Ley Orgánica 8/2013, Preámbulo IV)

La educación es la clave de esta transformación, mediante la formación de personas activas, con autoconfianza, curiosas, emprendedoras e innovadoras, deseosas de participar en la sociedad a la que pertenecen, de crear valor individual y colectivo, capaces de asumir como propio el valor del equilibrio entre el esfuerzo y la recompensa. (Ley Orgánica 8/2013, Preámbulo IV)

La LOMCE identifica la desigualdad con la ausencia de equidad. Ya no se trata de igualar por abajo -tal y como el texto legal sugiere que se ha venido haciendo hasta ahora-, pues con ello se avala la "desidia» y la «mediocridad", sino de favorecer un orden en el cual cada quien obtenga lo que se merece en función de sus logros. Todo indica que la educación no puede ser confundida con la simple "escolarización», ni menos aún con un «derecho».

La fórmula utilizada -recompensa por rendimientoevoca la racionalidad de mercado contemporánea consagrando, al tiempo, su ampliación al espacio escolar en su conjunto. El fallo histórico de los anteriores paradigmas, sugiere el legislador, ha consistido en una concepción graciable de los derechos; esto es, como si se tratase de bienes otorgados sin sujeciones, compensaciones o sanciones. Frente a ello, la Ley enfatiza la reconfiguración de las relaciones educativas en términos de un intercambio de prestaciones y contraprestaciones: derechos por «rendimiento», "esfuerzo» y voluntad ${ }^{16}$.

Una vez despojado de sus propiedades sociales, solo quedan las capacidades, talentos y valores del individuo, sobre quien recae la responsabilidad de sus erróneas -o acertadas- elecciones, tanto como la exigencia de un cambio orientado a comportarse activamente en el mercado escolar y, por extensión,

16 Se pone aquí en evidencia, también, el modelo del bienestar "condicionado» ("Welfare Conditionality») con el que la ley conecta y en el que se inscribe. Dicho modelo, extendido a escala internacional y a la de los distintos ámbitos de gestión de las políticas (empleo, vivienda, educación... etc.), se basa antes en la demanda y promoción de cambios comportamentales hacia las poblaciones «beneficiarias», que en su protección frente a los riesgos sociales a los cuales se ven confrontadas -desempleo, vivienda... etc.- (Watts y Fitzpatrick 2018). 
en el laboral. Además, en la medida en que el «fracaso» -y el éxito- escolar se abstraen de los procesos y de las condiciones socio-estructurales que intervienen en su génesis, la institución educativa se libera: se desvincula, en primer término, del horizonte ideal -definitivamente desacreditado por ideológico- del combate contra las desigualdades, dejando atrás las premisas de compensación y pacificación de la sociedad. En segundo, se exime de toda implicación en su producción, actualización o reforzamiento.

El tratamiento que hace la LOMCE del fracaso y la excelencia transforma, mediante un juicio de orden moral, las demandas externas en retos personales, poniendo al individuo en el centro mismo de dichas exigencias de carácter moral ${ }^{17}$.

Detrás de los talentos de las personas están los valores que los vertebran, las actitudes que los impulsan, las competencias que los materializan y los conocimientos que los construyen. (Ley Orgánica 8/2013, Preámbulo I)

En resumen, se pone de manifiesto la producción discursiva de una figura -el educando- caracterizada por rasgos psicológicos y valores morales que, como tal, ha de ser objeto de un trabajo educativo destinado a producir una subjetividad que asuma e incorpore la autoexigencia, y la capacidad de movilización del individuo. Como enuncia la Ley: «Uno de los objetivos [...] es introducir nuevos patrones de conducta». (Ley Orgánica 8/2013, Preámbulo II)

\subsection{Dispositivos de SUBJETIVACIÓN}

Todos los estudiantes poseen talento, pero la naturaleza de este talento difiere entre ellos. (Ley Orgánica 8/2013, Preámbulo I)

Si bien la LOMCE afirma la universalidad del talento, reconoce su diversa cualidad entre los estudiantes. Una vez predicadas como atributos del sujeto, se desprende inmediatamente que al nuevo paradigma educativo le corresponde, antes que nada, la función de reconocer, orientar, encauzar y, eventualmente, potenciar las capacidades con vistas a desarrollar los distintos talentos individuales, entendidos como capital a invertir:

17 Igual que el tratamiento que hace del paro, la exclusión social o, al contrario, de la integración, la capacidad de competir y acceder a puestos de alta cualificación.
El reconocimiento de esta diversidad entre alumno o alumna en sus habilidades y expectativas es el primer paso hacia el desarrollo de una estructura educativa que contemple diferentes trayectorias. La lógica de esta reforma se basa en la evolución hacia un sistema capaz de encauzar a los estudiantes hacia las trayectorias más adecuadas a sus capacidades, de forma que puedan hacer realidad sus aspiraciones y se conviertan en rutas que faciliten la empleabilidad y estimulen el espíritu emprendedor. (Ley Orgánica 8/2013, Preámbulo I)

La diversidad de "talentos» y "valores», por tanto, constituye el objeto a detectar y con el que trabajar pedagógicamente. El reconocimiento de las diferencias individuales se traduce en un conjunto de mecanismos de evaluación (y autoevaluación), precoces y sostenidos en el tiempo; y en el diseño de una nueva estructura educativa, compuesta de distintos itinerarios que, a su vez, organizan trayectorias escolares particulares, relativamente excluyentes.

Emerge así un sistema tan flexible como diversos son los "talentos», "rendimientos» y compromisos de los alumnos.

Resulta relevante detenerse brevemente en el despliegue de dispositivos que implementa la LOMCE, que concebimos como una expresión de la extensión de la racionalidad de mercado a la vida escolar. Siguiendo el planteamiento de Lahire, a propósito de las nuevas pedagogías de la "autonomía», entendemos por dispositivo pedagógico «un conjunto relativamente coherente de prácticas, discursivas y no discursivas, de arquitecturas, de objetos o máquinas, que contribuyen a orientar las acciones individuales y colectivas en una dirección» (2006:304). Desde este punto de vista, consideramos los dispositivos implementados por la LOMCE en su capacidad de actuar sobre las acciones y sobre la subjetividad: en el sentido en que tienden a incitar o movilizar, a comprometer o afectar los recursos personales, y a implicar al sujeto en un sentido especifico, contribuyendo, en última instancia, a la interiorización de las normas y los valores constitutivos del nuevo espíritu emprendedor (Parajuá, Franzé y García 2017) ${ }^{18}$.

18 Siguiendo a Boltanski y Chiapello (2002), entendemos que el proceso capitalista/productivo -y por tanto su racionalidad- no se agota en las formas organizacionales o jurídico/contractuales, sino que requiere para ponerse en marcha alguna forma de relación subjetiva e implicación personal. Es por ello que, como señalan Laval y Dardot (2013), las trasformaciones contemporáneas de la escuela, conciernen a la vez a las estructuras 
En primer lugar, los contenidos y la didáctica dejan de ser ejes centrales en la actividad educativa, lo que pone en primera línea a las «competencias» llamadas «transversales»:

Las habilidades cognitivas, siendo imprescindibles, no son suficientes; es necesario adquirir desde edades tempranas competencias transversales, como el pensamiento crítico, la gestión de la diversidad, la creatividad o la capacidad de comunicar, y actitudes clave como la confianza individual, el entusiasmo, la constancia y la aceptación del cambio. La educación inicial es cada vez más determinante por cuanto hoy en día el proceso de aprendizaje no se termina en el sistema educativo, sino que se proyecta a lo largo de toda la vida de la persona. (Ley Orgánica 8/2013, Preámbulo IV)

En la Ley se simplifican los conocimientos y se amplifican las actitudes. La educación básica obligatoria se concentra en la adquisición de conocimientos instrumentales genéricos: lecto-escritura, matemáticas, habilidades comunicativas y nuevas tecnologías de la información. Las "competencias» así adquiridas se convierten en herramientas básicas que permiten otros aprendizajes. Lo que entronca con la idea reiterada del aprendizaje permanente, fuera del aula, es decir, a lo largo de la vida en función de los requerimientos imprevisibles y fluctuantes del mercado ${ }^{19}$. En dicho contexto, de continuo cambio de las exigencias del mercado y de las relaciones laborales, en la lógica de la Ley, no tendría mucho sentido poner excesivo acento en conocimientos específicos de otro orden. El papel del Estado -y del sistema educativo- no es ya el de proporcionar, prioritariamente, cualificaciones ligadas a oficios y a la cultura de las profesiones, sino el de crear las condiciones de empleabilidad.

En este sentido, estamos ante un paradigma que subordina aquel tipo de conocimientos, al estímulo de una subjetividad comprometida en la intensificación del esfuerzo, en la superación de obstáculos, en la confrontación con el riesgo y en la disposición al

y a las subjetividades.

19 Cabe remarcar que tales actitudes y competencias son concebidas como el núcleo esencial de la labor educativa, tanto en el marco de referencia europeo (Comisión Europea 2008) como en las directrices de la OCDE (2012). Organismo, este último, fundamental en el desarrollo de iniciativas y recomendaciones destinadas al buen gobierno de las políticas, locales e internacionales, en materia económica. cambio. De ahí se sigue fácilmente que las competencias transversales, que son actitudinales y hasta caracteriales ("auto confianza», "entusiasmo», "constancia», "aceptación del cambio», etc.), adquieran más relevancia que el currículum, que ha quedado reducido a su expresión más básica e instrumental.

Conviene destacar que aquellas competencias son "transversales», también en tanto que "formas de ser" directamente transferibles a distintos escenarios y situaciones. En este proyecto las actitudes del individuo son la base de la empleabilidad, no del empleo. Como señala Hirtt (2009), se siguen necesitando trabajadores altamente cualificados a la vez que poco cualificados, pero, a diferencia de la situación anterior, y en la medida en que la flexibilidad es una nueva condición para poder adaptarse al mercado de trabajo, las competencias y disposiciones no son las mismas que las esperadas de la mano de obra de otrora.

En segundo lugar, como hemos adelantado, en este contexto se implementa un proceso evaluativo continuo. El diseño de distintos itinerarios formativos ${ }^{20}$,

20 A partir del artículo 20 y hasta el 31 se presentan los distintos itinerarios posibles y las evaluaciones correspondientes al final de cada etapa. Así, en lo que se refiere a la Educación Primaria, los centros educativos realizarán una evaluación a todos los alumnos al finalizar el tercer curso. En esta evaluación se comprobará el grado de adquisición de las competencias básicas en comunicación lingüística y matemática. De resultar desfavorable, el equipo docente podrá adoptar las medidas excepcionales más adecuadas, que podrán incluir la repetición del tercer curso.

Tiene especial interés en esta etapa la atención personalizada de los alumnos y, sobre todo, la realización de diagnósticos precoces y el establecimiento de mecanismos de refuerzo para lograr el éxito escolar. Por otra parte, los alumnos realizarán una evaluación al finalizar el sexto curso, sin efectos académicos, en la que se comprobará el grado de adquisición de las competencias básicas y de cumplimiento de objetivos de la etapa, así como la viabilidad del tránsito del alumno por la siguiente etapa. El resultado de esta evaluación tendrá carácter informativo y orientador para los centros, el equipo docente, las familias y los alumnos.

En la Educación Secundaria se introducen otros elementos diversificadores de trayectorias a partir del tercer curso, cuando los estudiantes deben elegir cursar la asignatura de Matemáticas Orientadas a las Enseñanzas Aplicadas -con las que no se puede seguir la ruta de Bachillerato- o las Matemáticas Orientadas a las Enseñanzas Académicas -que sí habilitan para ese itinerario-. A partir de cuarto curso los estudiantes pueden seguir tres itinerarios básicos: el segundo ciclo de ESO Acadé- 
justificado en la diversidad de talentos y expectativas -«sueños»- del alumnado, están precedidos de «evaluaciones» e «informes diagnósticos» (3ㅇde primaria, final de primaria; final 1 o ciclo de la ESO; revalidas al final de la Eso y al concluir el Bachillerato). La LOMCE concibe tales evaluaciones como componentes formativos e informativos; su propósito es el de aportar claves a los alumnos y sus familias en su toma de decisiones sobre la base de los resultados obtenidos (Ley Orgánica 8/2013, Preámbulo, VII). Tal y como ocurre con el modelo de trabajador -empresario de sí mismo- se desplaza al individuo la asunción de riesgos, así como la apuesta en el diseño de su trayectoria, la gestión y promoción de su carrera personal.

Las principales críticas recibidas por la ley han puesto el foco, prioritariamente, en la dimensión evaluativa, argumentado el estrés al que se somete a los alumnos y el hecho de que se les propone escoger vías educativas, relativamente excluyentes, desde edades muy tempranas. Desde nuestra perspectiva, y sin dejar de acordar con tales cuestionamientos, consideramos que el trasfondo de estos dispositivos apunta, más bien, a incitar a la competencia y a la maximización de uno mismo en una constante superación de etapas, que trasciende al propio sistema educativo y define una vida social y laboral de manera similar.

De hecho, la propia Ley anuncia determinados riesgos que amenazan a «sociedades»-e individuos- si no se sigue este proceso:

La principal amenaza a la que se enfrentan las sociedades desarrolladas es la fractura del conocimiento, esto es la fractura entre los que disponen de los conocimientos, competencias y habilidades para aprender y hacer, y hacer aprendiendo, y los que quedan excluidos. (Ley Orgánica 8/2013, Preámbulo III)

No se trata, por tanto, de un dispositivo disciplinario a la vieja usanza, destinado solamente a corregir

mico, el segundo ciclo de ESO Aplicado, o la Formación Profesional de Grado medio. A estos tres habría que añadir una cuarta posibilidad que es la de ingresar, a partir de los 15 años, en los estudios de Formación Profesional Básica. Al finalizar el cuarto curso, los alumnos realizarán una evaluación por la opción de enseñanzas académicas o por la de enseñanzas aplicadas. Los alumnos podrán realizar la evaluación por cualquiera de las dos opciones con independencia de la que hayan cursado en cuarto curso. los comportamientos mediante el temor a la sanción. Entendemos que actúa, sobre todo, como un modo de subjetivación. Como hemos señalado en otro lugar (Parajuá, Franzé y García 2017), es en la confrontación permanente con uno mismo, a lo largo del desarrollo evaluativo, donde residen sus verdaderas claves. Junto a otros autores (Laval et al. 2012), consideramos que este dispositivo se orienta a confrontar al sujeto con su condición de auditable (Shore y Wright 2000; Strathern 2000): es decir, a cultivar la disposición a ser examinado a lo largo de toda la vida, a través del sometimiento a pruebas constantes, a la actualización de sus competencias y al imperativo sistemático de obtener y mostrar resultados. Se trata, pues, de producir un sujeto que experimente íntimamente y haga suya, la condición de la empleabilidad en contextos flexibles.

En todo caso, ya no se garantiza un proceso normal, común, tal y como al menos lo prometían los sistemas educativos anteriores, conocidos como «comprehensivos». Por el contrario, al igual que en los recorridos profesionales, que se tornan discontinuos, se exige al alumnado y a su familia que se haga cargo de su propio camino, que elija y afronte los riesgos en cada etapa, -asumiendo sus éxitos y fracasos- frente a las distintas vías que se le abren y que eventualmente se mueva entre ellas.

\section{CONCLUSIÓN}

Hay una conexión estrecha entre las Leyes de reforma laboral, de apoyo al emprendimiento y la LOMCE. Esta conexión la entendemos como una lógica que toma todo sentido en el contexto del neoliberalismo, la desaparición de un régimen laboral, y la necesidad social y económica de nuevos sujetos. En esta lógica, el escenario escolar que define la LOMCE se convierte él mismo en una suerte de laboratorio de experimentación de lo flexible, lo incierto, la competencia, los incentivos, los riesgos, y, por tanto, la maximización del sí mismo. La invitación permanente a los sujetos a descubrir, movilizar y trabajar sus competencias y actitudes, produce un valor que es el que requiere un mercado laboral diseñado como cambiante y flexible. Cualquier elemento constitutivo del "talento" del sujeto (valores éticos, emociones, destrezas y habilidades prácticas de todo orden) se transforma en un capital destinado a ser invertido en el ámbito laboral. Como también apuntan Crespo y Serrano (2011), estamos ante esce- 
narios (educativo, laboral, profesional) que reclaman a los sujetos una actitud de perpetua adaptabilidad y maleabilidad. Son expresiones de una cultura de la brevedad y de la reinvención constante, acorde con la diversificación de los tiempos sociales, que necesita y produce sujetos con trayectorias no lineales, desarticuladas e inciertas. Pero son también la manifestación de las transformaciones que se están produciendo en las definiciones de la ciudadanía y de los sujetos de derechos: las incertidumbres de tiempos, espacios y trayectorias, se refieren a una erosión de los sostenes públicos y colectivos de los sujetos políticos, y al desarrollo y promoción del individuo privado como único agente, inevitablemente frágil, de la vida política.

\section{BIBLIOGRAFÍA CITADA}

Alonso, Luis Enrique y Carlos J. Fernández Rodríguez. 2013. «EI imaginario managerial: el discurso de la fluidez en la sociedad económica». En Luis Enrique Alonso y Carlos J. Fernández Rodríguez Los discursos del presente. Un análisis de los imaginarios sociales contemporáneos: 55-91. Madrid: Siglo XXI.

Alonso, Luis Enrique y Carlos J. Fernández Rodríguez. 2018. Poder y sacrificio. Los nuevos discursos de la empresa. Madrid: Siglo XXI.

Balasz, Gabrielle y Michel Pialoux. 1996. "Crise du travail et crise du politique». Actes de la recherche en sciences sociales 114: 3-4.

Boltanski, Luc y Ève Chiapello. 2002. El nuevo espíritu del capitalismo. Madrid: Akal.

Bourdieu, Pierre. 1985. «El discurso "importante". Algunas reflexiones sociológicas sobre "Quelques remarques critiques á propos de "Lire le Capital'"», en Pierre Bourdieu ¿Qué significa hablar? Economía de los intercambios lingüísticos: 171-187. Madrid: Akal.

Bourdieu, Pierre. 1998. "Le néo-libéralisme, utopie (en voie de réalisation) d'une exploitation sans limites», en Pierre Bourdieu Contre-feux. Propos pour servir à la résistance contre I'invasion néo-libérale: 108-119. París: Liber-Raisons d'agir.

Brown, Wendy. 2015. El pueblo sin atributos. La secreta revolución del neoliberalismo. Barcelona: Malpaso Ediciones.

Castel, Robert. 2003. L'insécurité social. Qu'est-ce qu'être protégé? París: Seuil.

Castel, Robert. 2010. El ascenso de las incertidumbres. Trabajo, protecciones, estatuto del individuo. Buenos Aires: FCE.

Comisión Europea. 2008. «De nouvelles compétences pour de nouveaux emplois. Anticiper les compétences requises et adapter l'offre de compétences aux besoins du marché du travail». Luxembourg: Office des Publications de l'Union Européenne. Disponible en: <https://ec.europa.eu/commission/presscorner/detail/fr/MEMO_08_791>.
Cowan Jane, Dembour, Marie y Wilson Richard. 2001. «Introduction». En Jane Cowan, Marie Dembour y Richard Wilson (eds.), Culture and Rights. Anthropological Perspectives: 1-26. Sussex: University of Sussex.

Cowan, Jane. 2010. "Cultura y derechos después de 'Culture and Rights'». Revista de Antropología Social 19: 67-101.

Crespo Suárez, Eduardo y Amparo Serrano Pascual. 2011. «Regulación del trabajo y el gobierno de la subjetividad: la psicologización política del trabajo». En Anastasio Ovejero Bernal (coord.), Psicología social crítica: 246-263. Madrid: Biblioteca Nueva.

Dubet, François. 2008. «El declive y las mutaciones de la institución». En María Isabel Jociles y Adela Franzé (eds.), ¿Es la escuela el problema? Perspectivas socio-antropológicas de etnografía y educación: 217-238. Madrid: Trotta.

Dumont, Louis. 1970. «Introducción». En Louis Dumont Homo hierarchicus. Ensayo sobre el sistema de castas: 3-26. Madrid: Aguilar.

Fernández Rodríguez, Carlos Jesús. 2007. El discurso del management: tiempo y narración. Madrid: CIS.

Fernández Rodríguez, Carlos Jesús y Amparo Serrano Pascual (Coords.). 2014. El paradigma de la flexiguridad en las políticas de empleo españolas: un análisis cualitativo: 489-505. Madrid: CIS.

Foucault, Michel. 1969. L'archéologie du savoir. Paris: Gallimard.

Harvey, David. 1998. La condición de la postmodernidad. Investigación sobre los orígenes del cambio cultural. Barcelona: Amorrortu.

Hirtt, Nico. 2009. "L'approche par compétences: une mystification pédagogique». L'école démocratique, 39: 1-34. Disponible en: <www.skolo.org/IMG/pdf/APC_Mystification.pdf >.

Lahire, Bernard. 2006. El espíritu sociológico. Buenos Aires: Manantial

Laval, Christian, Francis Vergne, Pierre Clément y Guy Dreux. 2012. La nouvelle école capitaliste. París: La Découverte.

Laval, Christian y Pierre Dardot. 2013. La nueva razón del mundo. Barcelona: Gedisa.

Parajuá Navarrete, Daniel, Adela Franzé Mudanó, y Erika García González. 2017. «Tecnologías y dispositivos pedagógicos en la reforma educativa (LOMCE) española: hacia el sujeto emprendedor y las fronteras sociales». Cuadernos del Instituto Nacional de Antropología y Pensamiento Latinoamericano 26(2): 5-21. Disponible en: <https://revistas.inapl.gob.ar/ index.php/cuadernos/article/view/1016/762>.

OCDE. 2012. "How Well are Countries Educating Young People to the Level Needed for a Job and a Living Wage?» Education Indicators in Focus 7. Disponible en: <https://www. oecd-ilibrary.org/education/how-well-are-countries-educating-young-people-to-the-level-needed-for-a-job-and-a-living-wage_5k91d4fsqj0w-en>.

Serrano, Amparo. 2016. «Colonización política de los imaginarios del trabajo: La invención paradójica del emprendedor». En Gil Calvo, Enrique (coord.), Sociólogos contra el economicismo: 110-128. Madrid: Catarata. 
Serrano, Amparo, Carlos J. Fernández Rodríguez y Alba Artiaga Leiras. 2014. «Epílogo: la Reforma Laboral de 2012: a golpe de metáforas». En Fernández Rodríguez, Carlos Jesús y Serrano Pascual, Amparo (coords.), El paradigma de la flexiguridad en las políticas de empleo españolas: un análisis cualitativo: 489-505. Madrid: CIS.

Serrano, Amparo y Carlos J. Fernández Rodríguez. 2018. «De la metáfora del mercado a la sinécdoque del emprendedor: la reconfiguración política del modelo referencial de trabajador». Cuadernos de Relaciones Laborales 36(2): 207-224.
Shore, Cris y Susan Wright. 2000. "Coercive accountability: the rise of audit culture in higher education». En Marilyn Strathern (ed.), Audit Culture. Anthropological studies in accountability, ethics and the academy: 57-89. London: Routledge.

Strathern, Marilyn. 2000. "Introduction: New accountabilities». En Marilyn Strathern (ed.), Audit Culture. Anthropological Studies in Accountability, Ethics and the Academy: 1-18. London: Routledge.

Watts, Beth y Suzanne Fitzpatrick. 2018. Welfare Conditionality. London: Routledge. 